\title{
Gesticulator: A framework for semantically-aware speech-driven gesture generation
}

\author{
Taras Kucherenko \\ KTH, Stockholm, Sweden \\ tarask@kth.se
}

\author{
Patrik Jonell \\ KTH, Stockholm, Sweden \\ pjjonell@kth.se
}

\author{
Sanne van Waveren \\ KTH, Stockholm, Sweden \\ sannevw@kth.se
}

\author{
Gustav Eje Henter \\ KTH, Stockholm, Sweden \\ ghe@kth.se
}

\author{
Simon Alexanderson \\ $\mathrm{KTH}$, Stockholm, Sweden \\ simonal@kth.se
}

\author{
Iolanda Leite \\ KTH, Stockholm, Sweden \\ iolanda@kth.se
}

\author{
Hedvig Kjellström \\ $\mathrm{KTH}$, Stockholm, Sweden \\ hedvig@kth.se
}

\begin{abstract}
During speech, people spontaneously gesticulate, which plays a key role in conveying information. Similarly, realistic co-speech gestures are crucial to enable natural and smooth interactions with social agents. Current end-to-end co-speech gesture generation systems use a single modality for representing speech: either audio or text. These systems are therefore confined to producing either acoustically-linked beat gestures or semantically-linked gesticulation (e.g., raising a hand when saying "high"): they cannot appropriately learn to generate both gesture types. We present a model designed to produce arbitrary beat and semantic gestures together. Our deep-learning based model takes both acoustic and semantic representations of speech as input, and generates gestures as a sequence of joint angle rotations as output. The resulting gestures can be applied to both virtual agents and humanoid robots. Subjective and objective evaluations confirm the success of our approach. The code and video are available at the project page svito-zar.github.io/gesticulator.
\end{abstract}

\section{KEYWORDS}

Gesture generation; virtual agents; socially intelligent systems; cospeech gestures; multi-modal interaction; deep learning

\section{ACM Reference Format:}

Taras Kucherenko, Patrik Jonell, Sanne van Waveren, Gustav Eje Henter, Simon Alexanderson, Iolanda Leite, and Hedvig Kjellström. 2020. Gesticulator: A framework for semantically-aware speech-driven gesture generation. In Proceedings of the 2020 International Conference on Multimodal Interaction (ICMI '20), October 25-29, 2020, Virtual event, Netherlands. ACM, New York, NY, USA, 9 pages. https://doi.org/10.1145/3382507.3418815

\section{INTRODUCTION}

When speaking, people often spontaneously produce hand gestures, also referred to as co-speech gestures. These co-speech gestures can accompany the content of the speech - what is being said - on all levels, from partial word meanings to situation descriptions [25]

Permission to make digital or hard copies of all or part of this work for personal or classroom use is granted without fee provided that copies are not made or distributed for profit or commercial advantage and that copies bear this notice and the full citation on the first page. Copyrights for components of this work owned by others than ACM must be honored. Abstracting with credit is permitted. To copy otherwise, or republish, to post on servers or to redistribute to lists, requires prior specific permission and/or a fee. Request permissions from permissions@acm.org.

ICMI '20, October 25-29, 2020, Virtual event, Netherlands

(C) 2020 Association for Computing Machinery.

ACM ISBN 978-1-4503-7581-8/20/10 . \$ \$15.00

https://doi.org/10.1145/3382507.3418815

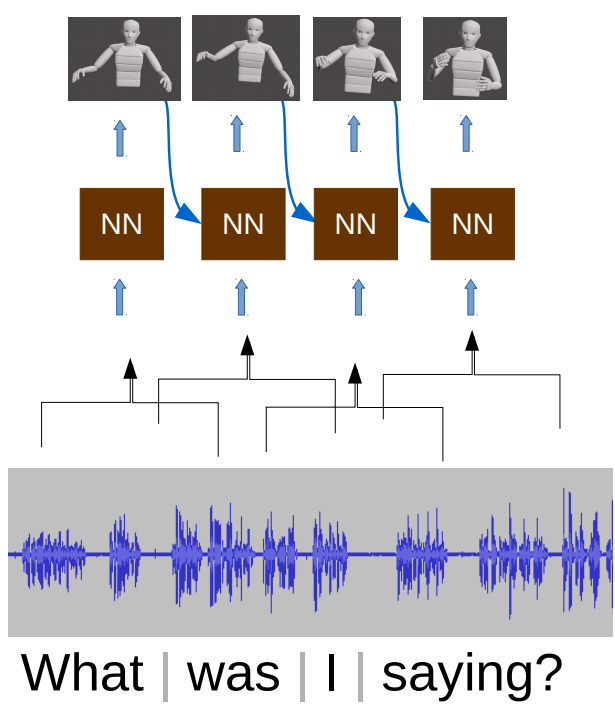

Figure 1: Overview of the proposed autoregressive model.

Gesture generation is hence an important part of animation, as well as of human-agent interaction research and applications.

Virtual agents have been developed for a diverse set of applications, such as serious gaming [32], interpersonal skills training $[35,45]$ or therapy systems [41]. Interactions with these virtual agents have shown to be more engaging when the agent's verbal behavior is accompanied by appropriate nonverbal behavior [43]. Moreover, it has been shown that manipulating gesture properties can influence user perception of an agent's emotions [7].

Traditionally, gesture generation for virtual agents has been done by various rule-based systems [6, 21, 44]. Those approaches are constrained by the discrete set of gestures they can produce. Alongside recent advances in deep learning, data-driven approaches have increasingly gained interest for gesture generation [1, 27, 48]. While early work has considered gesture generation as a classification task which aims to deduce a specified gesture class [9, 37], more recent work has considered it as a regression task which aims to produce continuous motion $[2,48]$. We focus on the latter task: continuous gesture generation. To date, prior work on continuous gesture generation has used a single input modality: either acoustic or semantic. In contrast, our work makes use of both these modalities to allow for semantic-aware speech-driven continuous gesture generation. The contributions of this work are the following: 
(1) the first data-driven model that maps speech acoustic and semantic features into continuous 3D gestures;

(2) a comparison contrasting the effects of different architectures and important modelling choices;

(3) objective and subjective evaluations of the effect of the two speech modalities - audio and semantics - on the resulting gestures.

We additionally extend a publicly available corpus of 3D cospeech gestures, the Trinity College dataset [13], with manual text transcriptions. Video samples from our evaluations are provided at vimeo.com/showcase/6737868.

\section{BACKGROUND AND RELATED WORK}

\subsection{Background}

While there are several theories on how gestures are produced by humans $[5,10,33]$, there is a consensus that speech and gestures correlate strongly [18, 23, 31, 39]. In this section, we review some concepts relevant to our work, namely gesture classification, the temporal alignment between gestures and speech as well as the gesture-generation problem formulation.

2.1.1 Co-Speech Gesture Types. Our work is informed by the gesture classification by McNeill [33], who distinguished the following gesture types:

(1) Iconic gestures represent some aspect of the scene;

(2) Metaphoric gestures represent an abstract concept;

(3) Deictic gestures point to an object or orientation;

(4) Beat gestures are used for emphasis and usually correlate with the speech prosody (e.g., intonation and loudness).

The first three gesture types, also called representational gestures, depend on the content of the speech - its semantics - while the last type instead depends on the audio signal - the acoustics. Hence, systems that ignore either aspect of speech can only learn to model a subset of human co-speech gesticulation.

2.1.2 Gesture-Speech Alignment. Gesture-speech alignment is an active research field covering several languages, including French [12], German [4], and English [18, 31, 39]. We focus on prior work on gesture-speech alignment for the English language.

In English, gestures typically lead the corresponding speech by, on average, $0.22 \mathrm{~s}$ (std $0.13 \mathrm{~s}$ ) [31]; specifically, Pouw et al. [39] aligned different gesture types with the peak pitch of the speech audio and found that the onset of beat gestures usually precedes the corresponding speech by $0.35 \mathrm{~s}$ (std 0.3 ), the onset of iconic gestures precedes speech by $0.45 \mathrm{~s}$ (std 0.4 ), and the onset of pointing gestures precedes speech by $0.38 \mathrm{~s}$ (std 0.4 ).

Informed by these works, we take the widest range among the studies, plus some margin, for the time-span of the speech used to predict the corresponding gesture, and consider $1 \mathrm{~s}$ of future speech and $0.5 \mathrm{~s}$ of past speech as input to our model detailed in Sec. 4.

2.1.3 The Gesture-Generation Problem. We frame the problem of speech-driven gesture generation as follows: given a sequence of speech features $s=\left[s_{t}\right]_{t=1: T}$ the task is to generate a corresponding pose sequence $\hat{\boldsymbol{g}}=\left[\hat{g}_{t}\right]_{t=1: T}$ of gestures that an agent might perform while uttering this speech. Here, $t=1: T$ denotes a sequence of vectors for $t$ in 1 to $T$.
Each speech segment $\boldsymbol{s}_{t}$ is represented by several different features, such as acoustic features (e.g., spectrograms), semantic features (e.g., word embeddings) or a combination of the two. The ground-truth pose $\boldsymbol{g}_{t}$ and the predicted pose $\hat{\boldsymbol{g}}_{t}$ at the same time instance $t$ can be represented in 3D space as a sequence of joint rotations: $\boldsymbol{g}_{t}=\left[\alpha_{i, t}, \beta_{i, t}, \gamma_{i, t}\right]_{i=1: n}, n$ being the number of keypoints of the body and $\alpha, \beta$ and $\gamma$ representing rotations in three axes.

\subsection{Related Work}

As this work contributes toward data-driven gesture generation, we confine our review to these methods.

2.2.1 Audio-Driven Gesture Generation. Most prior work on datadriven gesture generation has used the audio-signal as the only speech-input modality in the model $[14,15,19,28,42]$. For example, Sadoughi and Busso [42] trained a probabilistic graphical model to generate a discrete set of gestures based on the speech audiosignal, using discourse functions as constraints. Hasegawa et al. [19] developed a more general model capable of generating arbitrary 3D motion using a deep recurrent neural network, applying smoothing as postprocessing step. Kucherenko et al. [28] extended this work by applying representation learning to the human pose and reducing the need for smoothing. Recently, Ginosar et al. [15] applied a convolutional neural network with adversarial training to generate 2D poses from spectrogram features. However, driving either virtual avatars or humanoid robots requires 3D joint angles. Ferstl et al. [14] followed the approach of adversarial training and applied it to a recurrent neural network together with a gesture phase classifier. Our model differs from these systems in that it leverages both the audio signal and the text transcription for gesture generation.

2.2.2 Text-Transcription-Driven Gesture Generation. Several recent works mapped from text transcripts to co-speech gestures. Ishi et al. [22] generated gestures from text input through a series of probabilistic functions: Words were mapped to word concepts using WordNet [34], which then were mapped to a gesture function (e.g., iconic or beat), which in turn were mapped to clusters of 3D hand gestures. Yoon et al. [48] learned a mapping from the utterance text to gestures using a recurrent neural network. The produced gestures were aligned with audio in a post-processing step. Although these works capture important information from text transcriptions, they may fail to reflect the strong link between gestures and speech acoustics such as intonation, prosody, and loudness [40].

2.2.3 Multimodal Gesture-Generation Models. Only a handful of works have used multiple modalities of the speech to predict matching gestures. The model in Neff et al. [37] predicted gestures based on text, theme, rheme, and utterance focus. They also incorporated text-to-concept mapping. Concepts were then mapped to a set of 28 discrete gestures in a speaker-dependent manner. Chiu et al [9] used both audio signals and text transcripts as input, to predict a total of 12 gesture classes using deep learning. Our approach differs from these works, as we aim to generate a wider range of gestures: rather than predicting a discrete gesture class, our model produces arbitrary gestures as a sequence of $3 \mathrm{D}$ poses.

2.2.4 Regarding Motion Continuity. Separate from the input modalities of the system is the aspect of visual motion quality. Continuous 
gesture generation can avoid the concatenation-point discontinuities exhibited by playback-based approaches such as motion graphs $[3,26]$. That said, comparatively few approaches to continuous gesture generation explicitly try to enforce continuity in the generated pose sequence. Instead, they rely on postprocessing to increase smoothness as in [19]. Yoon et al. [48] include a velocity penalty in training that discourages jerky motion. The recurrent connections used in several models $[13,19,48]$ can also act as a pose memory that may help the model to produce smooth output motion. Autoregressive motion models have recently demonstrated promising results in probabilistic audio-driven gesture generation [2]. In this paper, we similarly investigate autoregressive connections for improving motion quality, which explicitly provide the most recent poses as input to the model when generating the next pose.

\section{TRAINING AND TEST DATA}

We develop our gesture generation model using machine learning: we learn a gesture estimator $\hat{\boldsymbol{g}}=F(\boldsymbol{s})$ based on a dataset of human gesticulation, where we have both speech information $s$ (acoustic and semantic) and gesture data $\boldsymbol{g}$. For this work, we specifically used the Trinity Gesture Dataset [13], comprising 244 minutes of audio and motion capture recordings of a male actor speaking freely on a variety of topics. We removed lower-body data, retaining 15 upper-body joints out of the original 69. Fingers were not modelled due to poor data quality.

To obtain semantic information for the speech, we first transcribed the audio recordings using Google Cloud automatic speech recognition (ASR), followed by thorough manual review to correct recognition errors and add punctuation for both the training and test parts of the dataset. The same data was used by the GENEA 2020 gesture generation challenge ${ }^{1}$ and has been made publicly available in the original dataset repository ${ }^{2}$.

\subsection{Test-Segment Selection}

Two 10-minute recordings from the dataset were held out from training. We selected 50 segments of $10 \mathrm{~s}$ for testing: 30 random segments and 20 semantic segments, in which speech and recorded gestures were semantically linked. Three human annotators marked time instants where the recorded gesture was semantically linked with the speech content. Instances where all three annotators agreed (within $5 \mathrm{~s}$ tolerance) were used as semantic segments in our experiments.

\subsection{Audio-Text Alignment}

Text transcriptions and audio typically have different sequence lengths. To overcome this, we encode words into frame-level features as illustrated in Figure 2. First, the sentence, excluding filler words, is encoded by BERT [11], which is the state-of-the-art model in natural language processing (NLP). We encode filler words and silence, which do not contain semantic information, as special, fixed vectors $V_{f}$ and $V_{s}$, respectively. Filler words typically indicate a thinking process and can occur with a variety of gestures. Therefore, we set the text feature vector $V_{f}$ during filler words equal to the average of the feature vectors for the most common filler words in the data. Silence typically has no gesticulation [17], so the silence

\footnotetext{
${ }^{1}$ genea-workshop.github.io/2020/\#gesture-generation-challenge

2 trinityspeechgesture.scss.tcd.ie
}

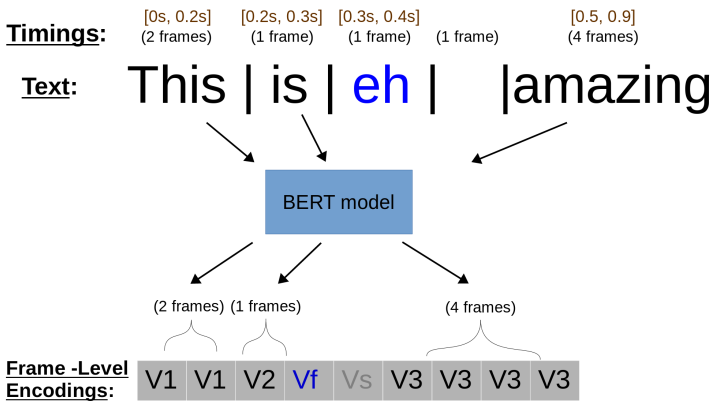

Figure 2: Encoding text as frame-level features. First, the sentence (omitting filler words) is encoded by BERT [11]. We thereafter repeat each vector according to the duration of the corresponding word. Filler words and silence are encoded as fixed vectors, here denoted $V f$ and Vs.

Table 1: Text and duration features for each frame.

BERT encoding of the current word

Time elapsed from the beginning of the word (in seconds)

Time left until the end of the word (in seconds)

Duration of this word (in seconds)

Relative progress through the word (in \%)

Speaking rate of this word (in syllables/second)

feature vector $V_{s}$ was made distinct from all other encodings, by setting all elements equal to -15 . Finally, we use timings from the ASR system to nonuniformly upsample the text features, such that both text and audio feature sequences have the same length and timings. This is a standard text-speech alignment method in the closely-related field of speech synthesis [47].

\section{SPEECH-DRIVEN GESTURE GENERATION}

This section describes our proposed method for generating upperbody motion from speech acoustics and semantics.

\subsection{Feature Types}

We base our features on the state of the art in speech audio and text processing. Throughout our experiments, we use frame-synchronized features with $20 \mathrm{fps}$.

Like previous research in gesture generation $[13,15]$, we represent speech audio by log-power mel-spectrogram features. For this, we extracted 64-dimensional acoustic feature vectors using a window length of $0.1 \mathrm{~s}$ and hop length $0.05 \mathrm{~s}$ (giving $20 \mathrm{fps}$ ).

For semantic features, we use BERT [11] pretrained on English Wikipedia: each sentence of the transcription is encoded by BERT resulting in 768 features per word, aligned with the audio as described in Sec. 3.2. We supplement these by five frame-wise scalar features, listed in Table 1.

To extract motion features, the motion-capture data was downsampled to $20 \mathrm{fps}$ and the joint angles were converted to an exponential map representation [16] relative to a T-pose; this is common in computer animation. We verified that the resulting features did 
not contain any discontinuities. Thereafter, we reduced the dimensionality by applying PCA and keeping $92 \%$ of the variance of the training data, similar to [48]. This resulted in 12 components.

\subsection{Model Architecture and Training}

We believe that a simple model architecture is preferable to a more complex one, everything else being equal. Hence, the intent of this work was to develop a straightforward model that solves the studied task. Figure 3 illustrates our model architecture. First, the text and audio features of each frame are jointly encoded by a feed-forward neural network to reduce dimensionality. To provide more input context for predicting the current frame, we pass a sliding window spanning $0.5 \mathrm{~s}$ (10 frames) of past speech and $1 \mathrm{~s}$ (20 frames) of future speech features over the encoded feature vectors. These time spans are grounded in research on gesture-speech alignment, as reviewed in Sec. 2.1.2. The encodings inside the context window are concatenated into a long vector and passed through several fully-connected layers. The model is also autoregressive: we feed preceding model predictions back to the model as can be seen in the figure, to ensure motion continuity. To condition on the information from the previous poses, we use FiLM conditioning [38], which generalizes regular concatenation. FiLM applies element-wise affine transforms $\operatorname{FiLM}(x, \boldsymbol{\alpha}, \boldsymbol{\beta})=x * \boldsymbol{\alpha}+\boldsymbol{\beta}$ to network activations $\boldsymbol{x}$, where scaling $\boldsymbol{\alpha}$ and offset $\boldsymbol{\beta}$ vectors are produced by a neural net taking other information (here previous poses) as input. The final layer of the model and of the conditioning network for FiLM are linear to not restrict the attainable output range.

\subsection{Training Procedure}

We train our model on sequences of aligned speech audio, text, and gestures from the dataset. Each training sequence contains 70 consecutive frames from a larger recording. The first 10 and the last 20 frames establish context for the sliding window, while the 40 central frames are used for training. The model is optimized end-to-end for 100 epochs using stochastic gradient descent (SGD) and Adam [24] to minimize the loss function $\operatorname{loss}(\boldsymbol{g}, \hat{\boldsymbol{g}})=$ $\operatorname{MSE}(\boldsymbol{g}, \hat{\boldsymbol{g}})+\lambda \operatorname{MSE}(\Delta \boldsymbol{g}, \Delta \hat{\boldsymbol{g}})$, here $\boldsymbol{g}$ and $\Delta \boldsymbol{g}$ are the ground-truth position and velocity, $\hat{\boldsymbol{g}}$ and $\Delta \hat{\boldsymbol{g}}$ are the same quantities for the model prediction and MSE stands for Mean Squared Error. The weight $\lambda$ was set empirically to 0.6 . Our velocity penalty can be seen as an improvement on the penalty used by Yoon et al. [48] Instead of penalizing the absolute value of the velocity, we enforce velocity to be close to that of the ground truth.

During development, we observed that information from previous poses (the autoregression) tended to overpower the information from the speech: our initial model moved independently of speech input and quickly converged to a static pose. This is a common failure mode in generative sequence models, cf. [8, 20]. To counteract this, we pretrain our model without autoregression for the first seven epochs (a number chosen empirically), before letting the model receive autoregressive input. This pretraining helps the network learn to extract useful features from the speech input, an ability which is not lost during further training. Additionally, while full training begins without any teacher forcing (meaning that the model receives its own previous predictions as autoregressive input instead of the ground-truth poses), this is annealed over time: after

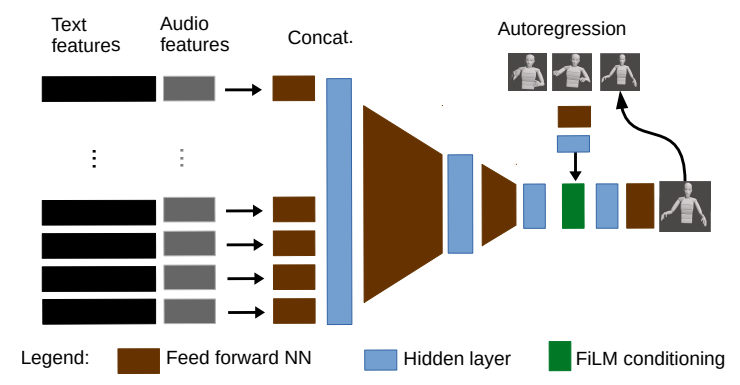

Figure 3: Our model architecture. Text and audio features are encoded for each frame and the encodings concatenated. Then, several fully-connected layers are applied. The output pose is fed back into the model in an autoregressive fashion.

one epoch, the model receives the ground-truth poses instead of its own prediction (for two consecutive frames) every 16 frames, which increased to every eight frames after another epoch, to every four frames after the next epoch, and then to every single frame after that. Hence, after five epochs of training with autoregression, our model has full teacher forcing: it always receives the groundtruth poses for autoregression. This procedure greatly helps with learning a model that properly integrates non-autoregressive input.

\subsection{Hyper-Parameter Settings}

For the experiments in this paper, we used the hyper-parameter search tool Tune [30]. We performed random search over 600 configurations with velocity loss as the only criterion, obtaining the following hyper-parameters: Speech-encoding dimensionality 124 at each of 30 frames, producing 3720 elements after concatenation. The three subsequent layers had 612, 256, and 12 or 45 nodes (the output dimensionality with or without PCA). Three previous poses were encoded into a 512-dimensional conditioning vector. The activation function was tanh, the batch size was 64 and the learning rate $10^{-4}$. For regularization, we applied dropout with probability 0.2 to each layer, except for the pose encoding, which had dropout 0.8 to prevent the model from attending too much to past poses.

\section{EVALUATION MEASURES}

In this section we describe the objective and subjective measures we used in our experiments (Secs. 6 and 7).

\subsection{Objective Measures}

There is no consensus in the field about which objective measures should be used to evaluate the quality of generated gestures. As a step towards common evaluation measures for the gesture generation field, we primarily use metrics proposed by previous researchers. Specifically, we evaluated the average values of rootmean-square error (RMSE), acceleration and jerk (rate of change of acceleration), and acceleration histograms of the produced motion, in line with Kucherenko et al. [28]. To obtain these statistics, the gestures were converted from joint angles to $3 \mathrm{D}$ joint positions.

The acceleration and jerk were averaged over all frames for all 14 3D joints (except for the hips, which were fixed). To investigate the motion statistics in more detail, we also computed velocity histograms of the generated motion and compared those against 
histograms derived from the ground-truth test data. We calculated the relative frequency of different velocity values over time-frames in all 50 test sequences, split into bins of width $1 \mathrm{~cm} / \mathrm{s}$.

\subsection{Subjective Measures}

To investigate human perception of the gestures we conducted several user studies that all followed the same protocol and procedure.

5.2.1 Experiment Design. We assessed the perceived human-likeness of the virtual character's motion and how the motion related to the character's speech using measures adapted from recent co-speech gesture generation papers $[15,48]$. Specifically, we asked the questions "In which video...”: (Q1) “...are the character's movements most human-like?" (Q2) "...do the character's movements most reflect what the character says?" (Q3) "...do the character's movements most help to understand what the character says?" (Q4) "...are the character's voice and movement more in sync?"

We used attention checks to filter out inattentive participants. For four of the six attention checks, we picked a random video in the pair and heavily distorted either the audio (in the 2nd and 17 th video pairs) or the video quality (in the 7 th and 21 st video pairs). Raters were asked to report any video pairs where they experienced audio or video issues, and were automatically excluded from the study upon failing any two of these four attention checks. In addition, the 13th and 24th video pairs presented the same video (from the random pool) twice. Here an attentive rater should answer "no difference".

5.2.2 Experimental Procedure. Participants were recruited on Amazon Mechanical Turk (AMT) and assigned to one specific comparison of two systems; they could complete the study only once, and were thus only exposed to one system pair. Each participant was asked to evaluate 26 same-speech video pairs on the four subjective measures: 10 pairs randomly sampled from a pool of 28 random segments, 10 from a pool of 20 semantic segments, and 6 attention checks (see above). These video pairs were then randomly shuffled.

Every participant first completed a training phase to familiarize themselves with the task and interface. This training consisted of five items not included in the analysis, with video segments not present in the study, showing gestures of different quality. Then, during the experiment, the videos in each pair were presented side by side in random order and could be replayed as many times as desired. For each pair, participants indicated which video they thought best corresponded to a given question (one of Q1 through Q4 above), or that they perceived both videos to be equal in regard to the question.

\section{ABLATION STUDY}

In this section, we evaluate the importance of various model components by individually ablating them, training seven different system variants including the full model (see Table 2). Comparisons against other gesture-generation approaches are reported in Sec. 7.

\subsection{Objective Evaluation}

In this section we report objective metrics, as described in Sec. 5.1.

6.1.1 Average Motion Statistics. Table 3 illustrates acceleration and jerk, as well as RMSE, averaged over 50 test samples for the ground
Table 2: The seven system variants in the ablation study

\begin{tabular}{l|l}
\hline System & Description \\
\hline Full model & The proposed method \\
No PCA & No PCA is applied to output poses \\
No Audio & Only text is used as input \\
No Text & Only audio is used as input \\
No FiLM & Concatenation instead of FiLM \\
No Velocity loss & The velocity loss is removed \\
No Autoregression & The previous poses are not used
\end{tabular}

Table 3: Objective evaluation of our systems: mean and standard deviation over 50 samples.

\begin{tabular}{lrrc}
\hline System & Accel. $\left(\mathrm{cm} / \mathrm{s}^{2}\right)$ & Jerk $\left(\mathrm{cm} / \mathrm{s}^{3}\right)$ & RMSE $(\mathrm{cm})$ \\
\hline Full model & $37.6 \pm 4.3$ & $830 \pm 89$ & $11.4 \pm 11.8$ \\
\hline No PCA & $63.8 \pm 8.3$ & $1332 \pm 192$ & $13.0 \pm 14.7$ \\
No Audio & $26.9 \pm 3.9$ & $480 \pm 67$ & $11.3 \pm 11.7$ \\
No Text & $27.0 \pm 1.9$ & $715 \pm 63$ & $10.9 \pm 11.3$ \\
No FiLM & $44.2 \pm 6.6$ & $931 \pm 181$ & $11.0 \pm 11.5$ \\
No Velocity loss & $36.4 \pm 4.1$ & $779 \pm 93$ & $11.4 \pm 12.3$ \\
No Autoregression & $120.3 \pm 19.2$ & $3890 \pm 637$ & $11.2 \pm 12.0$ \\
\hline Ground truth & $\mathbf{1 4 4 . 7} \pm 36.6$ & $\mathbf{2 3 2 2} \pm 538$ & $\mathbf{0}$
\end{tabular}

truth and the different ablations of the proposed method. Groundtruth statistics are given as reference values for natural motion. We focus our analysis on the jerk, since it is commonly used to evaluate the smoothness of the motion: the lower the jerk the smoother the motion is $[36,46]$.

We can observe that the proposed model exhibits lower jerk than the original motion. This is probably because our model is deterministic and hence produces gestures closer to the mean pose. Not using PCA results in higher acceleration and jerk, and made the model statistics closer to the ground truth. Our intuition for this is that PCA reduced variability in the data, which resulted in oversmoothed motion. Removing either audio or text input reduced the jerk even further. This is probably because these ablations provide a weaker input signal to drive the model, making it gesticulate closer to the mean pose. Both FiLM conditioning and the velocity penalty seem to have little effect on the motion statistics and are likely not central to the model. That autoregression is a key aspect of our system is clear from this evaluation: without autoregression, the model loses continuity and generates motion with excessive jerk. RMSE appears to not be informative. This is expected since there are many plausible ways to gesticulate, so the minimum-expected-loss output gestures do not have to be close to our ground truth.

6.1.2 Motion Velocity Histograms. The values in Table 3 were averaged over all time-frames and over all joints. To investigate the motion statistics in more detail, we computed velocity histograms of the generated motion and compared those against histograms derived from the ground-truth test data. As previous work has shown that wrist histograms are more informative than histograms averaged over all joints [28], we consider only left and right wrist joints. 


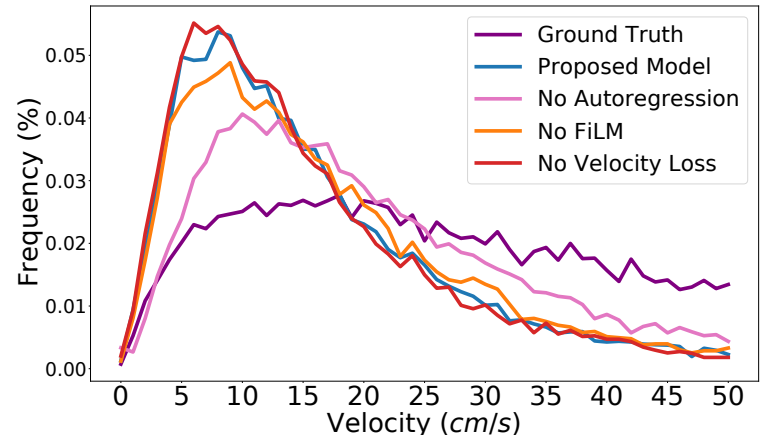

(a) Comparing different architectures.

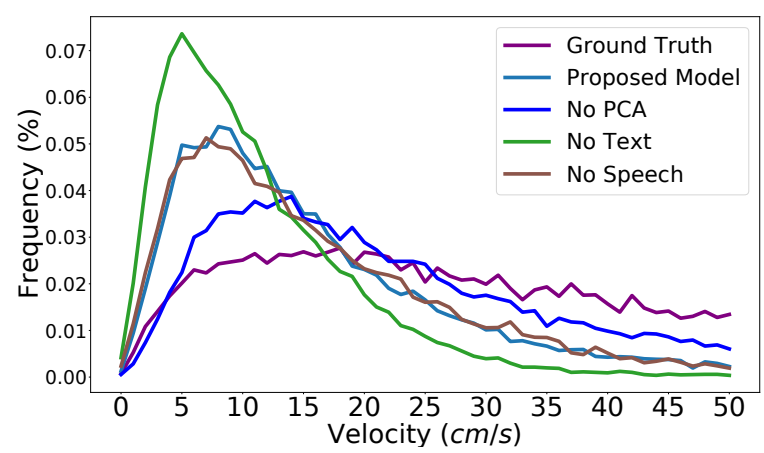

(b) Comparing different input/output data.

Figure 4: Velocity histograms of the wrist joints for the ablation study.

Figure 4a illustrates the velocity histogram of the wrist joints for the different model architectures and loss functions we considered. We observe two things: 1) the distributions are not influenced strongly by either FiLM conditioning or by velocity loss; and 2) autoregression reduces the amount of fast moments, making the velocity histogram more similar to the ground truth.

Velocity histograms for different input/output data are shown in Figure $4 \mathrm{~b}$. Removing PCA increases velocity, making the distribution more similar to the ground truth. In other words, training our model in the PCA space leads to reduced variability, which makes sense. We observe that excluding the text input makes the velocity smaller. This agrees with Table 3 and probably means that without semantic information the model produces mainly beat gestures, whose characteristics differ from other gesture types. While these numerical evaluations are valuable, they say very little about people's perceptions of the generated gestures.

\subsection{First Perceptual Study}

To investigate human perception of the gestures we conducted several user studies. This section reports on Perceptual Study 1, in which we evaluated participants' perception of a virtual character's gestures as produced by the seven variants of our model described in Table 2. The experimental procedure and evaluation measures (see Sec. 5.2) were identical across all perceptual studies, including this one. Video samples from all systems in this study can be found at vimeo.com/showcase/6737868.
In the comparison of system ablations (Perceptual Study 1), 123 participants ( $\mu$ age $=41.8 \pm 12.3 ; 52$ male, 70 female, 1 other) remained after exclusion of 477 participants who failed the attention checks, experienced technical issues, or stopped the study prematurely. The majority were from the USA $(N=120)$. Each sub-study had between 19 and 21 participants. We conducted a binomial test excluding ties with Holm-Bonferroni correction of $p$-values to analyze the responses. (24 responses that participants flagged for technical issues were excluded.) Our analysis was done in a double-blind fashion such that the conditions were obfuscated during analysis and only revealed to the authors after the statistical tests had been performed. The results are shown in Figure 5.

We can see from the evaluation of the "No Text" system that removing the semantic input drastically decreases both the perceived human-likeness of the produced gestures and how much they are linked to speech: participants preferred the full model over the one without text across all four questions asked with $p<.0001$. This confirms that semantics are important for appropriate automatic gesture generation.

The "No Audio" model is unlikely to generate beats, and might not follow an appropriate speech rhythm . Results in Figure 5 confirm this: participants preferred the full model over the one without audio across all four questions asked $(p<.0001)$.

Removing autoregression from the model only affected perceived naturalness, where it performed significantly worse $(p<.0001)$, as shown in Figure 5. This aligns with the findings from the objective evaluation: without autoregression the model produces jerky, unnatural-looking gestures, but the jerkiness does not influence whether gestures are semantically linked to the speech content.

There was no statistical difference between the Full model and the model without FiLM conditioning in terms of Q1 and Q2, but the model without FiLM was preferred with $p<.02$ for Q3 and $p<.04$ for Q4. This suggests that FiLM conditioning was not helpful for the model and regular concatenation worked better.

Removing the velocity penalty did not have a statistical difference on user responses, except for reducing user preference on Q4 with $p<.04$, suggesting that this component is not critical for the model.

The model without PCA gave unexpected results. In videos, we see that removing PCA improved gesture variability. While for human-likeness, there was no statistical difference, "No PCA" was significantly better $(p<.0001)$ on Q2, Q3 and Q4 (see Figure 5). In summary, participants preferred the system without PCA, so it was chosen as our final model for the remaining comparisons (in Sec. 7).

\subsection{Relation between objective and subjective evaluations}

Objective and subjective evaluations each have their pros and cons. In this subsection we analyze the empirical correlation between the two for the experiments reported here.

From, the "No FiLM" and "No Velocity Loss" conditions, we see that user ratings did not change much for ablations that only produced minor changes in motion statistics. This is not surprising. For models with low jerk compared to the ground truth, we can see that participants preferred the models where the jerk was closer to that of the ground truth motion ("No PCA"). However, too high jerk 

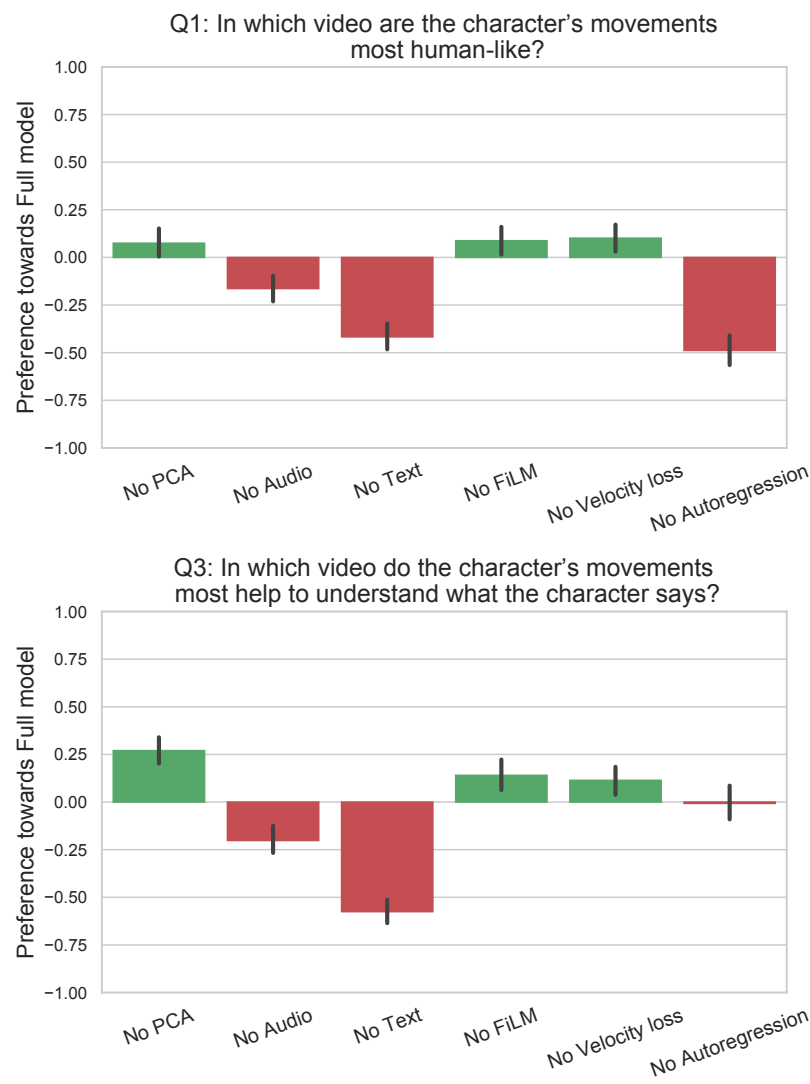
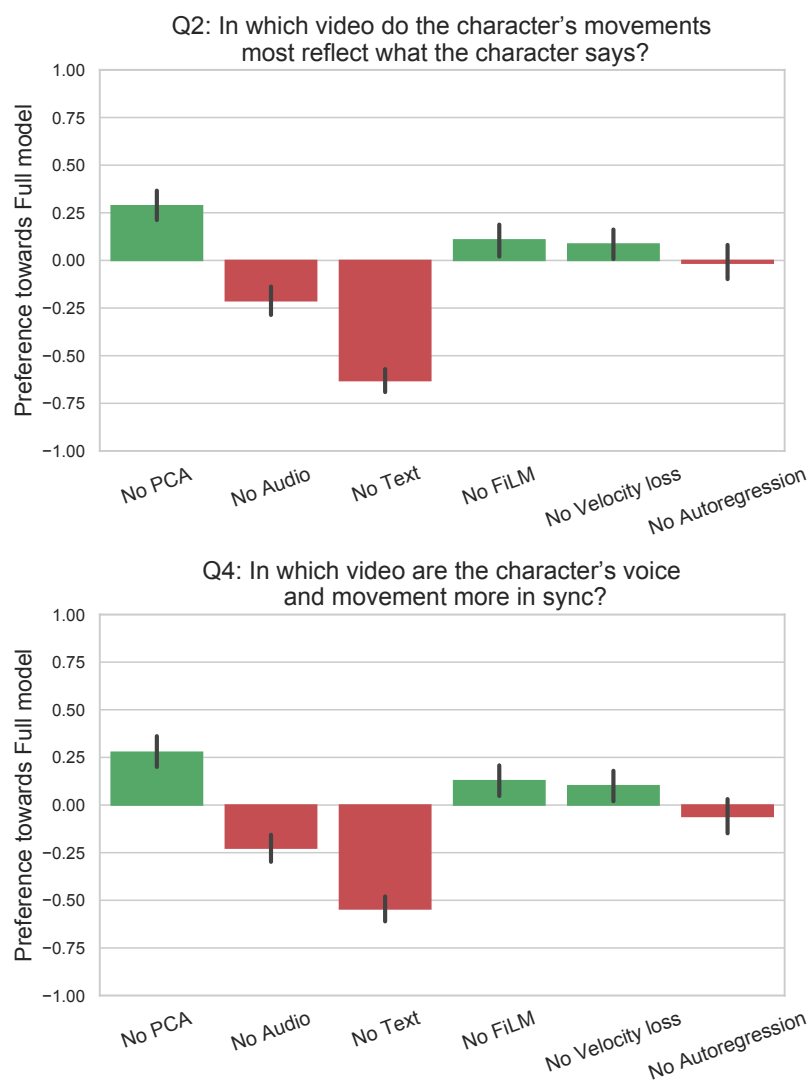

Figure 5: Results of Perceptual Study 1: comparing different ablations of our model in pairwise preference tests. Four questions, listed above each bar chart, were asked about each pair of videos. The bars show the preference towards the full model (higher values mean stronger preference) with $95 \%$ confidence intervals.

was associated with unnatural motion ("No Autoregression"). These results seem to indicate that jerk analysis provides information about the human-likeness of the motion.

\section{ADDITIONAL EVALUATIONS AND COMPARISONS}

The primary goal of this work is to develop the first model for continuous gesture generation that takes into account both the semantics and the acoustics of the speech. That said, we also benchmark our model against the state of the art in gesture generation. We compare the proposed approach to the model by Ginosar et al. [15], which is based on CNNs (convolutional neural networks) and GANs (generative adversarial networks), and therefore denoted CNN-GAN.

The hyper-parameters for the baseline method were fine-tuned by changing one parameter at a time and manually inspecting the visual quality of the resulting gestures on the validation dataset. The final hyper-parameters for the CNN-GAN [15] model were: batch size $=256$, number of neurons in the hidden layer $=256$, learning rate $=0.001$, training duration $=300$ epochs and $\lambda$ coefficient for the discriminator loss $=5$. This tuned system was compared against the best system ("No PCA") identified in Sec. 6.
Table 4: Objective comparison of our systems with the state-of-theart: mean and standard deviation over 50 samples

\begin{tabular}{lcc}
\hline System & Accel. $\left(\mathrm{cm} / \mathrm{s}^{2}\right)$ & Jerk $\left(\mathrm{cm} / \mathrm{s}^{3}\right)$ \\
\hline Final model (no PCA) & $63.8 \pm 8.3$ & $1330 \pm 192$ \\
CNN-GAN [15] & $254.7 \pm 31.8$ & $5280 \pm 631$ \\
\hline Ground truth & $144.2 \pm 35.9$ & $2315 \pm 530$
\end{tabular}

\subsection{Comparing with the state-of-the-art}

Like the previous experiments, we follow the objective evaluation setup described in Sec. 5.1. Table 4 displays the average acceleration and jerk over 50 test sequences. We observe that the proposed method has acceleration and jerk values roughly half of those exhibited by the ground truth, while the CNN-GAN [15] baseline instead has twice the acceleration and jerk of the ground truth.

To investigate which model is preferred by human observers, we conducted another user study. We evaluated participants' preference between the gestures as produced by the proposed models (No PCA) and CNN-GAN [15] (Perceptual Study 2). Video samples from this study can be found at vimeo.com/showcase/7127462. 


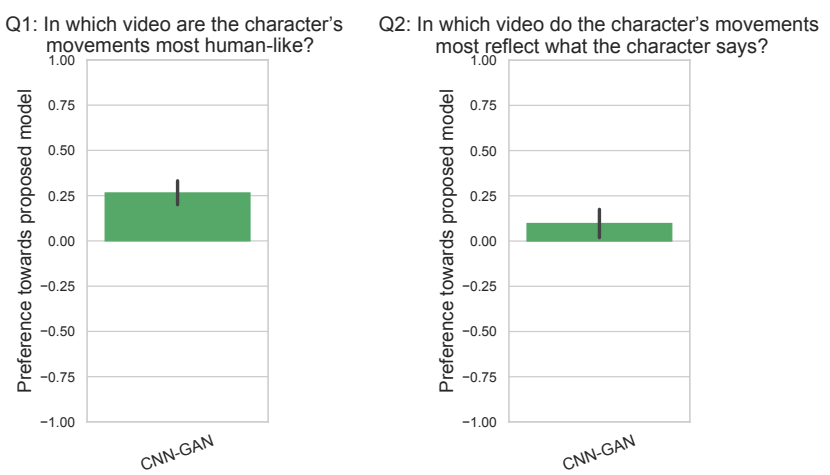

Figure 6: Results of Perceptual Study 2: comparing with the state-ofthe-art in pairwise preference tests. The bars show the preference towards the full model with $\mathbf{9 5 \%}$ confidence intervals.

The study setup was the same described in Sec. 5.2, except for three minor changes:

(1) We paid online participants more ( $5 \$$ instead of $3 \$$ ), since we realized that the effort required from participants in the previous study was higher than we had anticipated.

(2) We clarified the instructions for reporting broken audio/video.

(3) We only asked questions Q1 (human-likeness of movements) and Q2 (movements reflect what the character says).

In this study 27 participants $(\mu$ age $=41.7 \pm 11.3 ; 14$ male, 13 female) remained after exclusion of 43 participants based on the same criteria as before. The majority were from the USA $(N=25)$.

Like for Perceptual Study 1, we analyzed the responses using binomial tests excluding ties followed by Holm-Bonferroni correction. The results are shown in Figure 6. Our model was preferred over the CNN-GAN baseline for Q1 with $p<.0001$ and for Q2 with $p<.02$, indicating that the gestures generated by our model were perceived as more human-like and better reflected what the character said.

\subsection{Comparison with the Ground Truth}

We also compared our model to the ground-truth gestures using the same procedure as before. In this study (Perceptual Study 3), 20 participants ( $\mu$ age $=39.1 \pm 8.4 ; 9$ male, 11 female) remained after excluding 31 participants through the same criteria as in Perceptual Study $1 . N=18$ were from the USA. There was a very substantial preference for the ground-truth motion (between 84 and 93\%) across all questions. All differences were statistically significant according to Holm-Bonferroni-corrected binomial tests ignoring ties.

\subsection{What Do "Semantic" Gestures Even Mean?}

Finally, we evaluated if using text input helps our model to produce more semantically-linked gestures, such as iconic, metaphoric and diectic. To this end, we compared our best model (No PCA) with and without text information in the input: the first variant of this model received both audio and text, while the second one received only audio as input.

We asked three annotators to select which segments out of 50 test segments for both conditions that were semantically linked with the speech content. The annotators were all male and had an average age of 25.3 years. They were not aware of our research questions.

The results of this annotation were interesting and surprising: while all of them marked more gestures to be semantically linked with the speech content for the model that used text than the model without text ( 2 vs 0,21 vs 9 and 9 vs 4 ), they had very low agreement: Cronbach's alpha was below 0.5 . The low agreement on which segment were semantic indicates that it is very subjective which gestures should be classified as semantically linked, which makes this and any similar evaluation challenging.

\section{CONCLUSIONS AND FUTURE WORK}

We have presented a new machine learning-based model for cospeech gesture generation. To the best of our knowledge, this is the first data-driven model capable of generating continuous gestures linked to both the audio and the semantics of the speech.

We evaluated different architecture choices and compared our model to an audio-based state-of-the-art baseline using both objective and subjective measures. All the study materials are publicly available at figshare.com/projects/Gesticulator/87128. Our findings indicate that:

(1) Using both modalities of the speech - audio and text - can improve continuous gesture-generation models.

(2) Autoregressive connections, while not commonplace in contemporary gesture-generation models, can enforce continuity of the gestures, without vanishing-gradient issues and with few parameters to learn. We also described a training scheme that prevents autoregressive information from overpowering other inputs.

(3) PCA applied to the motion space (as used in [48]) can restrict the model by removing perceptually-important variation from the data, which may reduce the range of gestures.

(4) The gestures from our model were preferred over the CNNGAN [15] baseline by the study participants.

The main limitation of our work is that it requires an annotated dataset (with text transcriptions), which is labor-intensive. To overcome this, one could consider training the model directly on transcriptions from Automatic Speech Recognition.

Additionally, the vocabulary used in this dataset is sub-optimal. As we can see in the frequency table provided at tinyurl.com/y22h6rtt, out of the $50 \mathrm{k}$ total words there are 4230 unique words and the first 8 words account for $30 \%$ of all words spoken. This makes it challenging to learn semantic relations between gestures and text.

Future work also involves making the model stochastic (as in [2]), using larger datasets (such as [29]) and further improving the semantic coherence of the gestures, for instance by treating different gesture types separately.

\section{ACKNOWLEDGEMENT}

The authors would like to thank Andre Pereira, Federico Baldassarre and Marcus Klasson for helpful discussions. This work was partially supported by the Swedish Foundation for Strategic Research Grant No.: RIT15-0107 (EACare), by the Swedish Research Council projects 2017-05189 (CrowdVR) and 2018-05409 (StyleBot) and by the Wallenberg AI, Autonomous Systems and Software Program (WASP) funded by the Knut and Alice Wallenberg Foundation. 


\section{REFERENCES}

[1] Chaitanya Ahuja, Shugao Ma, Louis-Philippe Morency, and Yaser Sheikh. 2019 To react or not to react: End-to-end visual pose forecasting for personalized avatar during dyadic conversations. In Proceedings of the International Conference on Multimodal Interaction. 74-84.

[2] Simon Alexanderson, Gustav Eje Henter, Taras Kucherenko, and Jonas Beskow. 2020. Style-controllable speech-driven gesture synthesis using normalising flows. Computer Graphics Forum 39, 2 (2020), 487-496.

[3] Okan Arikan and David A. Forsyth. 2002. Interactive motion generation from examples. ACM Transactions on Graphics 21, 3 (2002), 483-490.

[4] Kirsten Bergmann, Volkan Aksu, and Stefan Kopp. 2011. The relation of speech and gestures: Temporal synchrony follows semantic synchrony. In Proceedings of the 2nd Workshop on Gesture and Speech in Interaction (GeSpIn 2011).

[5] Timothy W. Bickmore. 2004. Unspoken rules of spoken interaction. Commun. ACM 47, 4 (2004), 38-44.

[6] Justine Cassell, Hannes Högni Vilhjálmsson, and Timothy Bickmore. 2001. BEAT: The behavior expression animation toolkit. In Proceedings of the Conference on Computer Graphics and Interactive Techniques. ACM.

[7] Gabriel Castillo and Michael Neff. 2019. What do we express without knowing?: Emotion in Gesture. In Proceedings of the 18th International Conference on Autonomous Agents and MultiAgent Systems. International Foundation for Autonomous Agents and Multiagent Systems, 702-710.

[8] Xi Chen, Diederik P. Kingma, Tim Salimans, Yan Duan, Prafulla Dhariwal, John Schulman, Ilya Sutskever, and Pieter Abbeel. 2017. Variational lossy autoencoder. In Proceedings of the International Conference on Learning Representations.

[9] Chung-Cheng Chiu, Louis-Philippe Morency, and Stacy Marsella. 2015. Predicting co-verbal gestures: A deep and temporal modeling approach. In Proceedings of the International Conference on Intelligent Virtual Agents. Springer.

[10] Mingyuan Chu and Sotaro Kita. 2016. Co-thought and co-speech gestures are generated by the same action generation process. Fournal of Experimental Psychology: Learning, Memory, and Cognition 42, 2 (2016), 257.

[11] Jacob Devlin, Ming-Wei Chang, Kenton Lee, and Kristina Toutanova. 2018. BERT: Pre-training of deep bidirectional transformers for language understanding. Proceedings of the Conference of the North American Chapter of the Association for Computational Linguistics (2018).

[12] Gaëlle Ferré. 2010. Timing relationships between speech and co-verbal gestures in spontaneous French. In Language Resources and Evaluation, Workshop on Multimodal Corpora, Vol. 6. 86-91.

[13] Ylva Ferstl and Rachel McDonnell. 2018. Investigating the use of recurrent motion modelling for speech gesture generation. In Proceedings of the International Conference on Intelligent Virtual Agents. ACM.

[14] Ylva Ferstl, Michael Neff, and Rachel McDonnell. 2020. Adversarial gesture generation with realistic gesture phasing. Computers \& Graphics (2020).

[15] Shiry Ginosar, Amir Bar, Gefen Kohavi, Caroline Chan, Andrew Owens, and Jitendra Malik. 2019. Learning Individual Styles of Conversational Gesture. In Proceedings of the International Conference on Computer Vision and Pattern Recognition. IEEE.

[16] F. Sebastian Grassia. 1998. Practical parameterization of rotations using the exponential map. Journal of Graphics Tools 3, 3 (1998), 29-48.

[17] Maria Graziano and Marianne Gullberg. 2018. When speech stops, gesture stops: Evidence from developmental and crosslinguistic comparisons. Frontiers in Psychology (2018).

[18] Maria Graziano, Elena Nicoladis, and Paula Marentette. 2019. How referential gestures align with speech: Evidence from monolingual and bilingual speakers. Language Learning 70, 1 (2019), 266-304.

[19] Dai Hasegawa, Naoshi Kaneko, Shinichi Shirakawa, Hiroshi Sakuta, and Kazuhiko Sumi. 2018. Evaluation of speech-to-gesture generation using bi-directional LSTM network. In Proceedings of the International Conference on Intelligent Virtual Agents. ACM.

[20] Gustav Eje Henter, Simon Alexanderson, and Jonas Beskow. 2020. MoGlow: Probabilistic and controllable motion synthesis using normalising flows. ACM Transactions on Graphics 39, 4 (2020), 236:1-236:14. https://doi.org/10.1145/ 3414685.3417836

[21] Chien-Ming Huang and Bilge Mutlu. 2012. Robot behavior toolkit: Generating effective social behaviors for robots. In Proceedings of the International Conference on Human Robot Interaction (HRI '12). ACM/IEEE.

[22] Carlos T. Ishi, Daichi Machiyashiki, Ryusuke Mikata, and Hiroshi Ishiguro. 2018 A speech-driven hand gesture generation method and evaluation in android robots. IEEE Robotics and Automation Letters (2018).

[23] Jana M. Iverson and Esther Thelen. 1999. Hand, mouth and brain. The dynamic emergence of speech and gesture. Fournal of Consciousness Studies 6, 11-12 (1999) $19-40$.
[24] Diederik P Kingma and Jimmy Ba. 2015. Adam: A method for stochastic optimization. In Proceedings of the International Conference on Learning Representations.

[25] Stefan Kopp, Hannes Rieser, Ipke Wachsmuth, Kirsten Bergmann, and Andy Lücking. 2007. Speech-gesture alignment. In Proceedings of the Conference of the

International Society for Gesture Studies.
[26] Lucas Kovar, Michael Gleicher, and Fréderic Pighin. 2002. Motion graphs. ACM Transactions on Graphics 21, 3 (2002), 473-482.

[27] Taras Kucherenko. 2018. Data driven non-verbal behavior generation for humanoid robots. In ACM International Conference on Multimodal Interaction, Doctoral Consortium (Boulder, CO, USA) (ICMI '18). ACM, 520-523.

[28] Taras Kucherenko, Dai Hasegawa, Gustav E. Henter, Naoshi Kaneko, and Hedvig Kjellström. 2019. Analyzing Input and Output Representations for Speech-Driven Gesture Generation. In Proceedings of the International Conference on Intelligent Virtual Agents. ACM.

[29] Gilwoo Lee, Zhiwei Deng, Shugao Ma, Takaaki Shiratori, Siddhartha S Srinivasa, and Yaser Sheikh. 2019. Talking With Hands 16.2 M: A Large-Scale Dataset of Synchronized Body-Finger Motion and Audio for Conversational Motion Analysis and Synthesis.. In ICCV. 763-772.

[30] Richard Liaw, Eric Liang, Robert Nishihara, Philipp Moritz, Joseph E. Gonzalez, and Ion Stoica. 2018. Tune: A research platform for distributed model selection and training. arXiv preprint arXiv:1807.05118 (2018).

[31] Daniel P. Loehr. 2012. Temporal, structural, and pragmatic synchrony between intonation and gesture. Laboratory Phonology 3, 1 (2012), 71-89.

[32] Samuel Mascarenhas, Manuel Guimarães, Rui Prada, João Dias, Pedro A Santos, Kam Star, Ben Hirsh, Ellis Spice, and Rob Kommeren. 2018. A virtual agent toolkit for serious games developers. In Proceedings of the Conference on Computational Intelligence and Games (CIG). IEEE, 1-7.

[33] David McNeill. 1992. Hand and Mind: What Gestures Reveal about Thought. University of Chicago Press.

[34] George A. Miller. 1995. WordNet: a lexical database for English. Commun. ACM (1995).

[35] Shannon Monahan, Emmanuel Johnson, Gale Lucas, James Finch, and Jonathan Gratch. 2018. Autonomous agent that provides automated feedback improves negotiation skills. In Proceedings of the International Conference on Artificial Intelligence in Education. Springer, 225-229.

[36] Pietro Morasso. 1981. Spatial control of arm movements. Experimental brain research 42, 2 (1981), 223-227.

[37] Michael Neff, Michael Kipp, Irene Albrecht, and Hans-Peter Seidel. 2008. Gesture modeling and animation based on a probabilistic re-creation of speaker style. ACM Transactions on Graphics (2008).

[38] Ethan Perez, Florian Strub, Harm De Vries, Vincent Dumoulin, and Aaron Courville. 2018. FiLM: Visual reasoning with a general conditioning layer. In Proceedings of the AAAI Conference on Artificial Intelligence.

[39] Wim Pouw and James A. Dixon. 2019. Quantifying gesture-speech synchrony. In Proceedings of the Gesture and Speech in Interaction Workshop.

[40] Wim Pouw, Steven J. Harrison, and James A. Dixon. 2019. Gesture-speech physics: The biomechanical basis for the emergence of gesture-speech synchrony. Fournal of Experimental Psychology: General (2019).

[41] Lazlo Ring, Timothy Bickmore, and Paola Pedrelli. 2016. Real-time tailoring of depression counseling by conversational agent. Iproceedings 2, 1 (2016), e27.

[42] Najmeh Sadoughi and Carlos Busso. 2019. Speech-driven animation with meaningful behaviors. Speech Communication (2019).

[43] Maha Salem, Katharina Rohlfing, Stefan Kopp, and Frank Joublin. 2011. A friendly gesture: Investigating the effect of multimodal robot behavior in human-robot interaction. In Proceedings of the International Symposium on Robot and Human Interactive Communication. IEEE.

[44] Giampiero Salvi, Jonas Beskow, Samer Al Moubayed, and Björn Granström. 2009. SynFace: Speech-driven facial animation for virtual speech-reading support. fournal on Audio, Speech, and Music Processing (2009).

[45] William R Swartout, Jonathan Gratch, Randall W Hill Jr, Eduard Hovy, Stacy Marsella, Jeff Rickel, and David Traum. 2006. Toward virtual humans. AI Magazine 27, 2 (2006), 96-96.

[46] Yoji Uno, Mitsuo Kawato, and Rika Suzuki. 1989. Formation and control of optimal trajectory in human multijoint arm movement. Biological cybernetics 61, 2 (1989), 89-101.

[47] Zhizheng Wu, Oliver Watts, and Simon King. 2016. Merlin: An open source neural network speech synthesis system. In Proceedings of the ISCA Speech Synthesis Workshop.

[48] Youngwoo Yoon, Woo-Ri Ko, Minsu Jang, Jaeyeon Lee, Jaehong Kim, and Geehyuk Lee. 2019. Robots learn social skills: End-to-end learning of co-speech gesture generation for humanoid robots. In Proceedings of the International Conference on Robotics and Automation. IEEE. 